\title{
ON SUBMANIFOLDS ALL OF WHOSE GEODESICS ARE CIRCLES IN A COMPLEX SPACE FORM
}

\author{
By SAdAhiro MAEdA AND Noriaki SATO
}

\section{Introduction.}

First of all we recall the notion of circles in a Riemannian manifold $\tilde{M}$. A curve $x(t)$ of $\tilde{M}$ parametrized by arc length $t$ is called a circle, if there exists a field of unit vectors $Y_{t}$ along the curve which satisfies, together with the unit tangent vectors $X_{t}=\dot{x}(t)$, the differential equations: $\tilde{\nabla}_{t} X_{t}=k Y_{t}$ and $\tilde{\nabla}_{t} Y_{t}=-k X_{t}$, where $k$ is a positive constant and $\tilde{\nabla}_{t}$ denotes the covariant differentiation $\tilde{\nabla}$ with respect to $X_{t}$. Let $p$ be an arbitary point of $\tilde{M}$. For a pair of orthonormal vectors $X$ and $Y \in T_{p} \tilde{M}$ and for a given constant $k>0$, there exists a unique circle $x(t)$, defined for $t$ near 0 , such that

$$
x(0)=p, X_{0}=X \text { and }\left(\tilde{\nabla}_{t} X_{t}\right)_{t=0}=k Y .
$$

If $\tilde{M}$ is complete, $x(t)$ can be defined for $-\infty<t<+\infty$ (for details, see [9]).

Recently, Sakamoto [12] studied submanifolds all of whose geodesics are circles in a real space form $\tilde{M}$. A Riemannian manifold of constant curvature is called a real space form. He showed the following.

THEOREM 0.1 [12]. Let $M$ be a submanifold in a real space form $\tilde{M}$. Then the following three conditions are equivalent.

(I) The submanifold $M$ is nonzero isotropic and has parallel second fundamental form.

(II) Every geodesic in $M$ is a circle in $\tilde{M}$.

(III) The submanifold $M$ is planar geodesic and not totally geodesic.

In this paper, here and in the sequel, the conditions ( I ), (II) and (III) stand for those of Theorem 0.1 , unless otherwise stated.

Moreover, Sakamoto [12] has classified such submanifolds $M$ in the Euclidean sphere $S^{m}$. Of course, the above three conditions are not equivalent in the case that the ambient manifold $\tilde{M}$ is a complex space form. A Kaehler manifold of constant holomorphic sectional curvature is called a complex space form.

When the ambient manifold $\tilde{M}$ is a complex projective space, Pak [11] classified submanifolds under the condition (III), and Nomizu [8] and Naitoh [6] under the condition (I). Due to their works, we see that the condition (III) is

Received July 6, 1982 
essentially stronger than (I) in the case that $\tilde{M}$ is a complex projective space. In fact, the submanifolds $S^{1} \times S^{n}(n \geqq 1), S U(3) / S O(3), S U(3), S U(6) / S p(3), E_{6} / E_{4}$ do not satisfy the condition (III) but they satisfy (I).

We now remark that the following problem is still open: Classify all submanifolds satisfying the condition (II) in a complex projective space. In general, we note that the condition (I) always implies (II) regardless of the ambient manifold. Roughly speaking, we may see that (II) is close to (I) (see, Proposition 3.1). So, it is natural to consider the following problem: Is the condition (II) quite the same as (I) when $\tilde{M}$ is a complex space form?

The aim of this paper is to give an affirmative partial answer to the above problem. For this purpose, we recall the notion of $C R$-submanifolds of a Kaehler manifold. A submanifold $M$ of a Kaehler manifold $\tilde{M}$ is called a $C R$-submanifold if there exists on $M$ a $C^{\infty}$-holomorphic distribution $\mathscr{D}$ such that its orthogonal complement $\mathscr{D}^{\perp}$ is a totally real distribution, i. e., $J \mathscr{D}_{p}^{\perp} \subseteq T_{p}^{\perp} M$. Hereafter we shall study the problem for the class of $C R$-submanifolds in a complex space form. But we assert that this class is not small. In fact, all holomorphic submanifolds, totally real submanifolds and real hypersurfaces are necessarily $C R$-submanifolds.

Our result is as follows:

THEOREM. Let $M$ be a CR-submanifold in a complex space form $\tilde{M}(c)$ of constant holomorphic sectional curvature $c$. Then the following two conditions are equivalent.

( I ) The submanifold $M$ is nonzero isotropic and has parallel second fundamental form.

(II) Every geodesic in $M$ is a circle in $\tilde{M}(c)$.

The authors wish to express their hearty thanks to Professor K. Ogiue for his encouragement and help during the preparation of this paper.

\section{Preliminaries.}

Let $M$ be an $n$-dimensional submanifold of a complex $m$-dimensional Kaehler manifold $\tilde{M}$ with complex structure $J$ and Kaehler metric $g$. Let $\tilde{\nabla}$ (resp. $\nabla$ ) be the covariant differentiation on $\tilde{M}$ (resp. $M$ ). Then the second fundamental form $\sigma$ of the immersion is defined by $\sigma(X, Y)=\tilde{\nabla}_{X} Y-\nabla_{X} Y$, where $X$ and $Y$ are vector fields tangent to $M$. For a vector field $\xi$ normal to $M$, we write $\tilde{\nabla}_{X} \xi=-A_{\xi} X+D_{X} \xi$, where $-A_{\xi} X$ (resp. $D_{X} \xi$ ) denotes the tangential (resp. normal) component of $\tilde{\nabla}_{X} \xi$. The covariant derivative $\bar{\nabla} \sigma$ of the second fundamental form $\sigma$ is defined by

$$
\left(\bar{\nabla}_{X} \sigma\right)(Y, Z)=D_{X}(\sigma(Y, Z))-\sigma\left(\nabla_{X} Y, Z\right)-\sigma\left(Y, \nabla_{X} Z\right)
$$

for all vector fields $X, Y$ and $Z$ tangent to $M$. The second fundamental form $\sigma$ is said to be parallel if $\bar{\nabla} \sigma=0$. In particular, if $\sigma$ vanishes identically, $M$ is said to be a totally geodesic submanifold of $\tilde{M}$. The submanifold $M$ is called a 
holomorphic submanifold (resp. totally real submanifold) of $\tilde{M}$ if each tangent space of $M$ is mapped into the tangent space (resp. normal space) by the complex structure $J$.

O'Neill [10] defined a notion of isotropic immersions. An isotropic immersion is an isometric immersion such that all its normal curvature vectors have the same length at each point. Namely, the length of the normal curvature vector is a function on the submanifold. In particular, if the function is constant, then the immersion is said to be constant isotropic. A planar geodesic immersion is an isometric immersion such that every geodesic of $M$ is locally contained in a 2-dimensional totally geodesic submanifold in $\tilde{M}$.

Now we write the Gauss and Codazzi equations:

$$
\begin{gathered}
g(\tilde{R}(X, Y) Z, W)=g(R(X, Y) Z, W)+g(\sigma(X, Z), \sigma(Y, W)) \\
-g(\sigma(X, W), \sigma(Y, Z)) \\
\{\tilde{R}(X, Y) Z\}^{\perp}=\left(\bar{\nabla}_{X} \sigma\right)(Y, Z)-\left(\bar{\nabla}_{Y} \sigma\right)(X, Z),
\end{gathered}
$$

where $\tilde{R}$ (resp. $R$ ) denotes the curvature tensor for $\tilde{\nabla}$ (resp. $\nabla$ ).

In particular, the Riemannian curvature tensor $\tilde{R}$ of a real space form $\tilde{M}(c)$ of constant curvature $c$ is given by

$$
\tilde{R}(\tilde{X}, \tilde{Y}) \tilde{Z}=c\{g(\tilde{Y}, \tilde{Z}) \tilde{X}-g(\tilde{X}, \tilde{Z}) \tilde{Y}\}
$$

for all vector fields $\tilde{X}, \tilde{Y}$ and $\tilde{Z}$ tangent to $\tilde{M}(c)$. The Riemannian curvature tensor $\widetilde{R}$ of a complex space form $\tilde{M}(c)$ of constant holomorphic sectional curvature $c$ is given by

$$
\begin{aligned}
\tilde{R}(\tilde{X}, \tilde{Y}) \tilde{Z}= & \frac{c}{4}\{g(\hat{Y}, \tilde{Z}) \tilde{X}-g(\tilde{X}, \tilde{Z}) \tilde{Y}+g(J \tilde{Y}, \tilde{Z}) J \tilde{X} \\
& -g(J \tilde{X}, \tilde{Z}) J \tilde{Y}+2 g(\tilde{X}, J \tilde{Y}) J \tilde{Z}\}
\end{aligned}
$$

for all vector fields $\tilde{X}, \tilde{Y}$ and $\tilde{Z}$ tangent to $\tilde{M}(c)$.

Here, for later use, we recall the following

Proposition 1.1 [3]. Let $M$ be a submanfold of a non-flat complex space form $\tilde{M}(c)$. Then $M$ is a holomorphic or a totally real submanifold of $\tilde{M}(c)$ if and only if $M$ satisfies $\widetilde{R}(X, Y) Z \in T M$, where $\tilde{R}$ denotes the curvature tensor of $\tilde{M}(c)$ and all vector fields $X, Y$ and $Z$ are tangent to $M$.

\section{2. $C R$-submanifolds.}

First we recall a notion of $C R$-submanifolds $M$ of a Kaehler manifold $\tilde{M}$ (see, Bejancu [1]).

Definition 2.1. A submanifold $M$ of a Kaehler manifold $\tilde{M}$ is called a $C R$ submanifold if there exists a $C^{\infty}$-distribution $\mathscr{D}: p \rightarrow \mathscr{D}_{p} \subseteq T_{p} M$ on $M$ satisfying 
the following conditions: (a) $\mathscr{D}$ is holomorphic (i.e., $J \mathscr{D}_{p}=\mathscr{D}_{p}$ at each point $p \in M$ ), and (b) the complementary orthogonal distribution $\mathscr{D}^{\perp}: p \rightarrow \mathscr{D}_{p}^{\perp} \subseteq T_{p} M$ is totally real (i.e., $J D_{p}^{\perp} \subseteq T_{p}^{\perp} M$ at each point $p \in M$ ).

It follows from definition that all holomorphic submanifolds and totally real submanifolds are necessarily $C R$-submanifolds in a trivial sense. A $C R$-submanifold is said to be proper if it is neither holomorphic nor totally real. For example, any real hypersurface is a proper $C R$-submanifold.

Chen [2] defines $C R$-products.

Definition 2.2. A $C R$-submanifold $M$ of a Kaehler manifold $\tilde{M}$ is called a $C R$-product if it is locally a Riemannian product of a holomorphic submanifold and a totally real submanifold of $\tilde{M}$.

Very recently, Chen [2] gave examples of $C R$-products of a complex projective space. We denote by $P_{k}^{N}(\boldsymbol{C})$ the complex $N$-dimensional complex projective space with constant holomorphic sectional curvature $k$. We can construct many proper $C R$-products of a complex projective space as follows: Let $M_{1}$ (resp. $M_{2}$ ) be any holomorphic submanifold (resp. totally real submanifold) of $P_{c}^{m}(\boldsymbol{C})\left(\right.$ resp. $\left.P_{c}^{n}(\boldsymbol{C})\right)$. Then

$$
M_{1} \times M_{2} \subset P_{c}^{m}(\boldsymbol{C}) \times P_{c}^{n}(\boldsymbol{C}) \stackrel{S_{m, n}}{\longleftrightarrow} P_{c}^{m n+m+n}(\boldsymbol{C})
$$

is a proper $C R$-product. Here we define a mapping

by

$$
S_{m, n}: P_{c}^{m}(\boldsymbol{C}) \times P_{c}^{n}(\boldsymbol{C}) \subset P_{c}^{m n+m+n}(\boldsymbol{C})
$$

$$
\left(z_{0}, \cdots, z_{m} ; \eta_{0}, \cdots, \eta_{n}\right) \longmapsto\left(z_{0} \eta_{0}, \cdots, z_{\imath} \eta_{3}, \cdots, z_{m} \eta_{n}\right),
$$

where $\left(z_{0}, \cdots, z_{m}\right)$ (resp. $\left.\left(\eta_{0}, \cdots, \eta_{n}\right)\right)$ are the homogeneous coordinates of $P_{c}^{m}(\boldsymbol{C})$ (resp. $P_{c}^{n}(\boldsymbol{C})$ ). $S_{m, n}$ is a well-known Kaehler imbedding, which is called a Segre ımbedding.

In contrast to this fact, when the ambient manifold $\tilde{M}$ is a complex hyperbolic space, Chen [2] showed the following remarkable result.

TheOREM 2.1 [2]. There exists no proper CR-product in any complex hyperbolic space.

Finally we prepare two lemmas without proof in order to get our Theorem.

LEMMA 2.1 [2]. A CR-submanifold $M$ of a Kaehler manifold $\tilde{M}$ is a CRproduct if and only if $A_{J \mathscr{D}}{ }^{\perp} \mathscr{D}=0$, where $A_{\xi}$ is the second fundamental form with respect to $\xi$ so that $g\left(A_{\xi} X, Y\right)=g(\sigma(X, Y), \xi)$.

Lemma 2.2 [2]. Let $M^{n}$ be a proper $C R$-product in $P_{4}^{m}(\boldsymbol{C})$. Then

$$
\left\{\sigma\left(X_{\imath}, Z_{\alpha}\right)\right\} \quad \imath=1, \cdots, 2 h ; \alpha=1, \cdots, p \quad(2 h+p=n)
$$

are orthonormal vectors in $\nu\left(T^{\perp} M=J \mathscr{D}^{\perp} \oplus \nu\right)$, where $X_{1}, \cdots, X_{2 h}$ and $Z_{2}, \cdots, Z_{\text {g }}$ 
are orthonormal bases for $\mathscr{D}_{p}$ and $\mathscr{D}_{p}^{\frac{1}{p}}$, respectively.

\section{Some results on the condition (II).}

We get

Proposition 3.1. Let $M$ be a submanifold in a Riemannian manifold $\tilde{M}$. Then the following two conditions are equivalent.

(i) The submanifold $M$ is nonzero isotropic and the second fundamental form $\sigma$ of $M$ in $\tilde{M}$ satısfies $\left(\bar{\nabla}_{X} \sigma\right)(X, X)=0$ for all vector fields $X$ tangent to $M$.

(ii) Every geodesic in $M$ is a circle in $\tilde{M}$.

Proof. The following discussion is quite the same as in [8]. (i) $\Rightarrow$ (ii): We denote by $\tilde{\nabla}$ and $\nabla$ the Riemannian connections of $\tilde{M}$ and $M$, respectively. Put $\lambda=\|\sigma(X, X)\| /\|X\|^{2}$. Let $x(t)$ be any geodesic with unit tangent vector $X_{t}$. Then $\lambda$ is constant along $x(t)$, because, by the second assumption of (i), we have

$$
\begin{aligned}
X_{t}\left(\lambda^{2}\right) & =X_{t}\left(g\left(\sigma\left(X_{t}, X_{t}\right), \sigma\left(X_{t}, X_{t}\right)\right)\right) \\
& =2 g\left(\left(\bar{\nabla}_{t} \sigma\right)\left(X_{t}, X_{t}\right), \sigma\left(X_{t}, X_{t}\right)\right) \\
& =0 .
\end{aligned}
$$

Hence, $\lambda$ is locally constant, because the immersion is isotropic.

Put $Y_{t}=\frac{1}{\lambda} \sigma\left(X_{t}, X_{t}\right)$. It follows by the Gauss formula that

$$
\tilde{\nabla}_{t} X_{t}=\sigma\left(X_{t}, X_{t}\right)=\lambda Y_{t}
$$

Moreover we get

$$
\tilde{\nabla}_{t} Y_{t}=\tilde{\nabla}_{t}\left(\frac{1}{\lambda} \sigma\left(X_{t}, X_{t}\right)\right)=\frac{1}{\lambda} \tilde{\nabla}_{t}\left(\sigma\left(X_{t}, X_{t}\right)\right) .
$$

By virture of the Weingarten formula we see

$$
\tilde{\nabla}_{t} Y_{t}=\frac{1}{\lambda}\left(-A_{\sigma\left(X_{t}, X_{t}\right)} X_{t}+D_{t}\left(\sigma\left(X_{t}, X_{t}\right)\right)\right) .
$$

Since $M$ is an isotropic submanifold, we easily see that

$$
A_{\sigma\left(X_{t}, X_{t}\right)} X_{t}=\lambda^{2} X_{t}
$$

and again by the second assumption we get $D_{t}\left(\sigma\left(X_{t}, X_{t}\right)\right)=0$.

Thus we see that every geodesic in $M$ is a circle in $\tilde{M}$. (ii) $\Rightarrow(\mathrm{i})$ : see, [8].

Q.E.D.

As an immediate consequence of Proposition 3.1, we find the following.

Remark 3.1. The condition ( I ) always implies (II) regardless of the ambient manifold $\tilde{M}$. 
Finally we investigate the condition $\left(\bar{\nabla}_{X} \sigma\right)(X, X)=0$

Proposition 3.2. Let $M$ be a submanifold of a complex space form $\tilde{M}(c)$ with complex structure $J$. Then the following two conditions are equivalent.

(A) $\left(\bar{\nabla}_{X} \sigma\right)(X, X)=0$ for all vector fields $X$ tangent to $M$.

(B) $\left(\bar{\nabla}_{X} \sigma\right)(Y, Z)=(c / 4)\{g(X, J Y) J Z+g(X, J Z) J Y\}^{\perp}$ for all vector fields $X, Y$ and $Z$ tangent to $M$, where $\{*\} \perp$ means the normal component of $\{*\}$.

Proof. Now we may easily see that $\left(\bar{\nabla}_{X} \sigma\right)(X, X)=0$ is equivalent to

$$
\left(\bar{\nabla}_{X} \sigma\right)(Y, Z)+\left(\bar{\nabla}_{Y} \sigma\right)(Z, X)+\left(\bar{\nabla}_{Z} \sigma\right)(X, Y)=0 .
$$

On the other hand, by the Codazzi equation (1.2) and (1.4), we get

$$
\begin{aligned}
\left(\bar{\nabla}_{X} \sigma\right) & (Y, Z)-\left(\bar{\nabla}_{Y} \sigma\right)(X, Z) \\
\quad & \{\tilde{R}(X, Y) Z\} \\
& =(c / 4)\{g(J Y, Z) J X-g(J X, Z) J Y+2 g(X, J Y) J Z\}^{\perp} .
\end{aligned}
$$

Exchanging $Y$ and $Z$, we have

$$
\begin{aligned}
& \left(\bar{\nabla}_{X} \sigma\right)(Z, Y)-\left(\bar{\nabla}_{Z} \sigma\right)(X, Y) \\
& \quad=\{\tilde{R}(X, Z) Y\}^{\perp} \\
& \quad=(c / 4)\{g(J Z, Y) J X-g(J X, Y) J Z+2 g(X, J Z) J Y\}^{\perp} .
\end{aligned}
$$

Summing up (3.1), (3.2) and (3.3), we obtain (B).

The converse is trivial.

Q.E.D.

Remark 3.2. When the ambient manifold $\tilde{M}$ is a real space form, $\left(\bar{\nabla}_{X} \sigma\right)(Y, Z)$ is symmetric for $X, Y$ and $Z$ from the Codazzi equation (1.2) and (1.3). Thus we easily see that $\left(\bar{\nabla}_{X} \sigma\right)(X, X)=0$ is equivalent to $\bar{\nabla} \sigma \equiv 0$. On the other hand, when the ambient manifold $\tilde{M}$ is a complex space form, this is not true. In fact, Chen and Vanhecke [4] proved that any small geodesic hypersphere in a complex space form satisfies the condition $\left(\bar{\nabla}_{X} \sigma\right)(X, X)=0$. It is well known that, as a consequence of the Codazzi equation (1.2), there does not exist a real hypersurface of a complex space form with parallel second fundamental form (for details, see [5]).

\section{Proof of Theorem.}

We have already seen that the condition ( I ) implies (II) by Proposition 3.1. In this section, we shall show that (II) implies (I) for $C R$-submanifolds. So, our aim here is only to prove that $\left(\bar{\nabla}_{X} \sigma\right)(Y, Z)$ is symmetric for $X, Y$ and $Z$ under the condition (II) again by Proposition 3.1. Namely, we have only to show that all $C R$-submanifolds of a complex space form with condition (II) are 
necessarily holomorphic submanifolds or totally real submanifolds (see, Proposition 1.1 and the Codazzi equation (1.2)). The following discussion consists of two parts. First, we shall prove that any $C R$-submanifold of a complex space form with condition (II) is a $C R$-product. Secondly, we shall show that any proper $C R$-product of a complex space form can't satisfy the condition (II).

We are now in a position to prove the following:

Proposition 4.1. Let $M$ be a CR-submanifold of a complex space form $\tilde{M}(c)$ with condition (II). Then $M$ is a CR-product in $\tilde{M}(c)$.

Proof. By the assumption, we decompose $T_{p} M$ into $\mathscr{D}_{p} \oplus \mathscr{D}_{p}^{\perp}$. Because of Lemma 2.1, it suffices to show that $A_{J \mathscr{D}}{ }^{\perp} \mathscr{D}=0$. First we shall check $g\left(A_{J} \mathscr{D}^{\perp} \mathscr{D}\right.$, $\mathscr{D})=0$, i.e., $g\left(\sigma(\mathscr{D}, \mathscr{D}), J \mathscr{D}^{\perp}\right)=0$. By Proposition 3.1, the immersion is $\lambda$-isotropic, that is,

$$
g(\sigma(X, X), \sigma(X, X))=\lambda^{2} g(X, X) g(X, X)
$$

holds for all vector fields $X$ tangent to $M$. We may easily see that (4.1) is equivalent to

$$
\begin{gathered}
g(\sigma(X, Y), \sigma(Z, W))+g(\sigma(X, Z), \sigma(Y, W))+g(\sigma(X, W), \sigma(Y, Z)) \\
=\lambda^{2}\{g(X, Y) g(Z, W)+g(X, Z) g(Y, W)+g(X, W) g(Y, Z)\} .
\end{gathered}
$$

For simplicity, in the following calculation, we may assume that $\nabla X=\nabla Y=$ $\nabla Z=\nabla W=0$ at a fixed point $p$ of $M$. Differentiating (4.2) with respect to $U$, we get

$$
\begin{aligned}
& g\left(\left(\bar{\nabla}_{U} \sigma\right)(X, Y), \sigma(Z, W)\right)+g\left(\sigma(X, Y),\left(\bar{\nabla}_{U} \sigma\right)(Z, W)\right) \\
& +g\left(\left(\bar{\nabla}_{U} \sigma\right)(X, Z), \sigma(Y, W)\right)+g\left(\sigma(X, Z),\left(\bar{\nabla}_{U} \sigma\right)(Y, W)\right) \\
& \quad+g\left(\left(\bar{\nabla}_{U} \sigma\right)(X, W), \sigma(Y, Z)\right)+g\left(\sigma(X, W),\left(\bar{\nabla}_{U} \sigma\right)(Y, Z)\right) \\
& =0
\end{aligned}
$$

at $p$, since $\lambda$ is locally constant (see the proof of proposition 3.1).

If, in particular, $X \in \mathscr{D}_{p}^{\perp}: Y, Z, U, W \in \mathscr{D}_{p}$ in (4.3), then we get from Proposition 3.2

$$
\begin{aligned}
g(U, J Y) g(J X, \sigma(Z, W)) & +g(U, J Z) g(J X, \sigma(Y, W)) \\
& +g(U, J W) g(J X, \sigma(Y, Z))=0 .
\end{aligned}
$$

Putting $U=J W$ in (4.4), we have

$$
\begin{aligned}
g(Y, W) g(J X, \sigma(Z, W)) & +g(W, Z) g(J X, \sigma(Y, W)) \\
& +g(W, W) g(J X, \sigma(Y, Z))=0 .
\end{aligned}
$$


Here we fix $Y$ and $Z$ in (4.5). Then, in the case of $\operatorname{dim} \mathscr{D}>2$, choosing $W(\neq 0)$ in such a way that $g(Y, W)=g(W, Z)=0$ at $p$, we get by (4.5)

$$
g(J X, \sigma(Y, Z))=0 \quad \text { at } p .
$$

This implies that $g\left(\sigma(\mathscr{D}, \mathscr{D}), J \mathscr{D}^{\perp}\right)=0$ in the case of $\operatorname{dim} \mathscr{D}>2$.

Next we shall check the case of $\operatorname{dim} \mathscr{D}=2$. Putting $Z=Y$ and choosing $W(\neq 0)$ in such a way that $g(Y, W)=0$ in (4.5), we get

$$
g(J X, \sigma(Y, Y))=0 \quad \text { at } p .
$$

On the other hand, putting $Z=J Y=J W$ in (4.5) we get

$$
g(J X, \sigma(Y, J Y))=0 \quad \text { at } p .
$$

Taking account of (4.6), (4.7) and (4.8), we find $g\left(\sigma(\mathscr{D}, \mathscr{D}), J \mathscr{D}^{\perp}\right)=0$.

Secondly, we shall check $g\left(A_{J} \mathscr{D}^{\perp} \mathscr{D}, \mathscr{D}^{\perp}\right)=0$, i.e., $g\left(\sigma\left(\mathscr{D}, \mathscr{D}^{\perp}\right), J \mathscr{D}^{\perp}\right)=0$. We now put $X, Z \in \mathscr{D}^{\perp} ; Y, U, W \in \mathscr{D}$ in (4.3). We get from Proposition 3.2

$$
\begin{aligned}
& g(U, J Y) g(J X, \sigma(Z, W))+g(U, J W) g(\sigma(X, Y), J Z) \\
& \quad+g(U, J W) g(J X, \sigma(Y, Z))+g(U, J Y) g(\sigma(X, W), J Z)=0 .
\end{aligned}
$$

In particular, putting $U=W=J Y$ in (4.9) and using (3.3) in Lemma 3.1 [2], we have

$$
g(\sigma(X, W), J Z)=0
$$

that is,

which implies

$$
g\left(A_{J Z} W, X\right)=0
$$

$$
g\left(A_{J \mathscr{D}} \perp \mathscr{D}, \mathscr{D}^{\perp}\right)=0
$$

Q.E.D.

Remark 4.1. It follows from Theorem 2.1 and Proposition 4.1 that our Theorem holds in the case that $\tilde{M}(c)$ is a complex hyperbolic space.

Finally we will prove our Theorem when the ambient manifold $\tilde{M}$ is a complex projective space. We get the following

Proposition 4.2. Let $M$ be a proper $C R$-product in $P^{m}(\boldsymbol{C})$. Then $M$ is not an isotropic submanifold.

Proof. We assume that there exists an isotropic proper $C R$-product in $P^{m}(\boldsymbol{C})$. Without loss of generality, $P^{m}(\boldsymbol{C})$ is equipped with the Fubini-Study metric of constant holomorphic sectional curvature 4. Let $M=M_{1}^{2 h} \times M_{2}^{p}$ be a proper $C R$ product (i.e., $h \neq 0$ and $p \neq 0$ ). We here denote by $M_{1}^{2 h}$ (resp. $M_{2}^{p}$ ) a $2 h$-dimensional holomorphic submanifold (resp. $p$-dimensional totally real submanifold) of $P^{m}(\boldsymbol{C})$. Let $\left\{X_{2}\right\}$ (resp. $\left.\left\{Z_{\alpha}\right\}\right)$ be an orthonormal basis on $M_{1}^{2 h}$ (resp. $M_{2}^{p}$ ). From (1.1), (1.4) and Lemma 2.2 we get 


$$
g\left(\sigma\left(X_{i}, X_{\imath}\right), \sigma\left(Z_{\alpha}, Z_{\alpha}\right)\right)=0 .
$$

On the other hand, using (4.2) we get

$$
2 g\left(\sigma\left(X_{\imath}, Z_{\alpha}\right), \sigma\left(X_{\imath}, Z_{\alpha}\right)\right)+g\left(\sigma\left(X_{\imath}, X_{\imath}\right), \sigma\left(Z_{\alpha}, Z_{\alpha}\right)\right)=\lambda^{2} .
$$

Here, again by Lemma 2.2 , we have

$$
g\left(\sigma\left(X_{\imath}, X_{\imath}\right), \sigma\left(Z_{\alpha}, Z_{\alpha}\right)\right)=\lambda^{2}-2 .
$$

Combining (4.10) and (4.11), we obtain $\lambda=\sqrt{2}$.

Thus we may find that $M_{1}$ is holomorphically immersed $\sqrt{ } 2$-isotropic submanifold in $P_{4}^{m}(\boldsymbol{C})$. This implies that $M_{1}$ is locally isometric to the Euclidean space $\boldsymbol{R}^{2 h}$. In fact, for any vector field $X$ tangent to $M_{1}$, by the Gauss equation (1.1) we have

$$
\begin{aligned}
g(R(X, J X) J X, X) & =4+g(\sigma(J X, J X), \sigma(X, X))-\|\sigma(J X, X)\|^{2} \\
& =4-2\|\sigma(X, X)\|^{2} \\
& =4-2 \lambda^{2} \\
& =0 .
\end{aligned}
$$

However, $\boldsymbol{R}^{2 h}$ can't be immersed holomorphically into $P^{m}(\boldsymbol{C})$ (see, [7]). This is a contradiction.

Q.E.D.

\section{REFERENCES}

[1] A. Bejancu, $C R$-submanifolds of a Kaehler manifold I., Proc. Amer. Math. Soc. 69 (1978), 134-142.

[2] B. Y. Chen, $C R$-submanifolds of a Kaehler manifold I., J. Diff. Geom. 16 (1981), 305-322.

[3] B. Y. Chen and K. Ogiue, On totally real submanıfolds, Trans. Amer. Math. Soc. 193 (1974), 257-266.

[4] B. Y. Chen and L. VAnnecke, Differential geometry of geodesic spheres, J. Reine Angew. Math. 325 (1981), 28-67.

[5] Y. MaEdA, On real hypersurfaces of a complex projective space, J. Math. Soc. Japan, 28 (1976), 529-540.

[6] H. NAITOH, Isotropic submanifolds with parallel second fundamental form in $P^{m}(c)$, Osaka J. Math. 18 (1981), 427-464.

[7] H. Nakagawa and K. Ogiue, Complex space forms immersed in complex space forms, Trans. Amer. Math. Soc. 219 (1976), 289-297.

[8] K. Nomizu, A characterization of the Veronese varieties, Nagoya Math. J. 60 (1976), 181-188.

[9] K. Nomizu and K. Yano, On circles and spheres in Riemannian Geometry, Math. Ann. 210 (1974), 163-170.

[10] B. O'NeILL, Isotropic and Kaehler immersions, Canad. J. Math. 17 (1965), 905-915.

[11] J.S. PAK, Planar geodesic submanifolds in complex space forms, Kodai Math. J. 
1 (1978), 187-196.

[12] K. Sakamoto, Planar geodesic immersions, Tohoku Math. J. 29 (1977), 25-56.

Department of Mathematics

Tokyo Metropolitan University

TOKYO, 158 JAPAN 